\title{
LA-UR- $98-3366$
}

Approved for public release; distribution is unlimited.

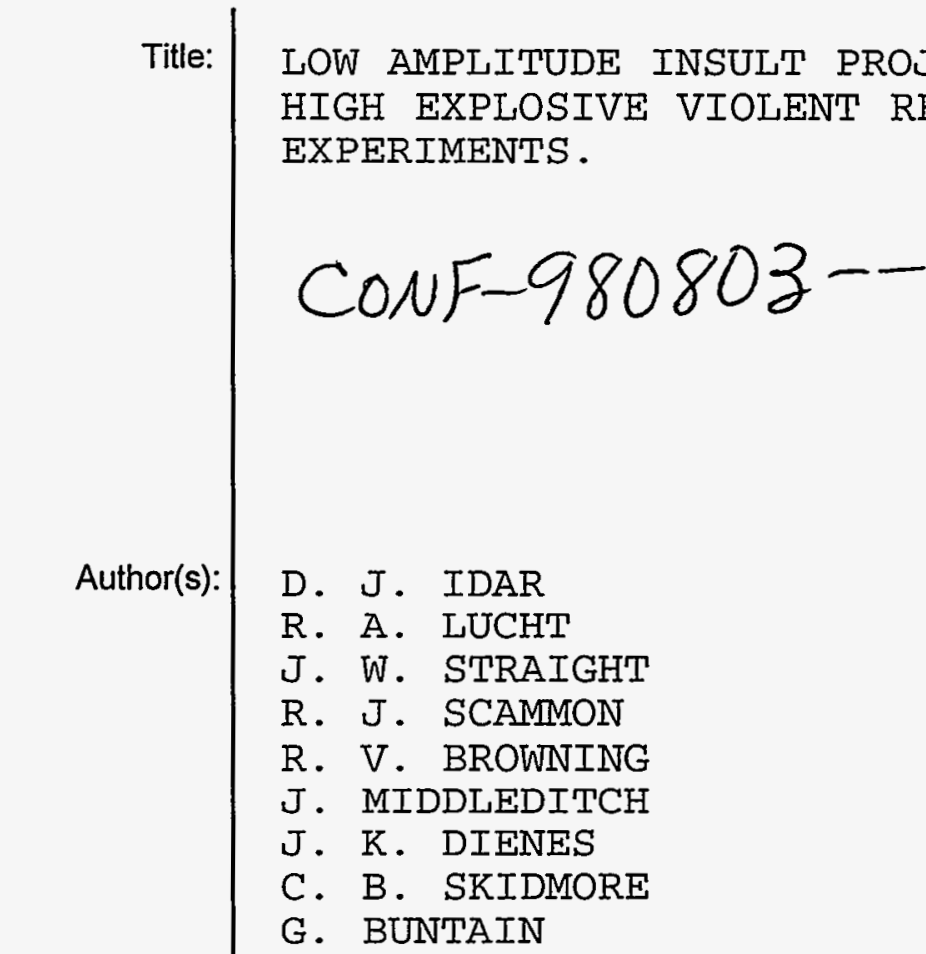

Submitted to

ELEVENTH INTERNATIONAL DETONATION SYMPOSIUM

SNOWMASS CONFERENCE CENTER, AUGUST 31 SEPTEMBER 4, 1998 SNOWMASS, COLORADO

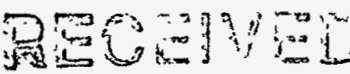

APR I 39999

30<smiles>CCCCCCCCCCCC</smiles>

\section{LOS Alamos}

Los Alamos National Laboratory, an affirmative action/equal opportunity employer, is operated by the University of California for the U.S. Department of Energy under contract W-7405-ENG-36. By acceptance of this article, the publisher recognizes that the U.S. Government retains a nonexclusive, royalty-free license to publish or reproduce the published form of this contribution, or to allow others to do so, for U.S. Government purposes. Los Alamos National Laboratory requests that the publisher identify this article as work performed under the auspices of the U.S. Department of Energy. The Las Alamos National Laboratory strongly supports academic freedom and a researcher's right to publish; as an institution, however, the Laboratory does not endorse the viewpoint of a publication or guarantee its technical correctness. 


\section{DISCLAIMIER}

This report was prepared as an account of work sponsored by an agency of the United States Government. Neither the United Stales Government aor any ageacy thereof, nor any of their employees, makes any warranty, express or implied, or essumes any legal liability or responsibility for the scevragy, compleseness, or usefulness of any information, apparatus, product, of process diselosed, of represents that its use would not infringe privately owned rights. Refereece berein to any specifie commercial produce, process, or serviee by trade anme, tradernart, inanuiase turer, or otherwise does aol necesearify constivute ar ingly its eadorsemeat, recom. mendation. or favoring by the United States Goyernmeat or any ageacy thereof. The views and opinions of authors expressed herein do not necessarily state or reflect those of the United States Goverameat or any agency thereof. 


\section{DISCLAIMER}

Portions of this document may be illegible in electronic image products. Images are produced from the best available original document. 


\title{
LOW AMPLITUDE INSULT PROJECT: PBX 9501 HIGH EXPLOSIVE VIOLENT REACTION EXPERIMENTS
}

\author{
D. J. Idar, R. A. Lucht, J. W. Straight, R. J. Scammon, R. V. Browning, \\ J. Middleditch, J. K. Dienes, C. B. Skidmore, and G. A. Buntain \\ Los Alamos National Laboratory \\ Los Alamos, NM 87545
}

\begin{abstract}
The Modified Steven test geometry has been used with several different target designs to investigate the mechanical loading behavior of PBX9501 to a low velocity impact. A $2 \mathrm{~kg}$. mild steel spigot projectile is launched via a new powder driven gun design, from $\sim 20$ to $105 \mathrm{~m} / \mathrm{s}$, at lightly confined, steel targets. Brief descriptions of the gun design and operation are given. The threshold velocity to reaction for various target designs, different PBX 9501 lots, and different high explosive (HE) thicknesses are reported and compared. Various diagnostics have been employed to evaluate the pressure profile and timing, and target strain behavior relative to projectile impact. The violence of reaction, as measured by both passive and active techniques, is reported relative to a steady state detonation in PBX 9501. Experimental results suggest slightly different ignition mechanisms dominate based on (HE) thickness, resulting in delayed reactions from $0.2-$ to 2.8 ms after impact. Post-test analyses of the PBX 9501 are briefly summarized.
\end{abstract}

\section{INTRODUCTION}

Low-velocity, mechanical impact followed by unintentional high explosive (HE) reaction are of concern in credible accident scenarios involving the handling, transport, and storage of energetic materials. The viability of these various scenarios have been evaluated with different experimental methods and techniques, from small- to large-scale, including, but not limited to the drop weight impact, Taylor anvil impact, Susan, and more recently the Steven tests. Ideally the experimental measurements will be used in the further development of reliable 3D predictive capabilities for the accurate assessment of safety scenarios.

The challenges in this type of research are numerous and complex. The HE formulations not only vary in composition, but also in binder type and percentage, density, porosity, and particulate distributions. These differences directly affect the $\mathrm{HE}$ material properties behavior and degree of sensitivity. Material confinement and impactor characteristics also play a significant role in the coupled behavioral response of the $\mathrm{HE}$ and the target to this stimulus.

Clues to mechanical loading ignition mechanisms can be found dispersed through the energetic material literature describing ingeniously devised experiments coupled with or without computational analyses to support the proposed mechanisms. Yet, none of these experiments or analyses has been sufficiently clever to account for all of the parameters that can affect the potential reaction sequence induced by mechanical impact in accident scenarios. The range and type of experiments are too multiple and diverse to cover briefly in the limited scope of this paper. However, its important to note that determining the best method to extrapolate the data to different dimension and mass scales further complicates data interpretation and usefulness. The key is to determine which are the most relevant parameters leading to ignition/reaction with 1) a sensitive, reproducible test configuration and reliable diagnostic measurements in tandem with computational analyses, and 2) a review of the appropriate literature for an evaluation of significant effects.

Our overall project objectives are to 1) determine and evaluate the reaction threshold behavior of the $\mathrm{HE}$, 2) assess the ignition/reaction profile induced in the $\mathrm{HE}$ by the mechanical loading event, i.e. deformation, time, pressure, and temperature history, and ignition locus(ci) as a function of the pertinent parameters, and 3) develop a reliable 3-D computational predictive capability. This paper summarizes our to-date experimental test methods and results with the HMXbased formulation, PBX 9501, in modified Steven target configurations. New spigot gun design and operation characteristics are described. Threshold determinations, pressure measurements, timing, relative reaction levels, $\mathrm{HE}$ aging, and $\mathrm{HE}$ thickness parameters with corresponding results are also described. Further research work is still required to meet our experimental and computational objectives.

\section{EXPERIMENTAL}

For. this research work, several types of test arrangements, targets, and diagnostics have been used. Each will be described in different sections. 


\section{SPIGOT GUN DESIGN, MOUNT, AND CHARACTERIZATION TESTS}

The projectile characteristics required were a mass of $2 \mathrm{~kg}$ of arbitrary size, shape, and material travelling at velocities between 22 and $105 \mathrm{~m} / \mathrm{s}$. The arbitrary size and shape limited the utility of a conventional projectile-in-tube launch system, which would require an elaborate sabot design and a massive sabot stripper. Although a gas driven system would have worked well for the slower projectile velocities, it would not be practical for the higher ones.

For these reasons, a powder gun using rifle powder and driving a spigot projectile, with the head external to the barrel and a shank extending inside the barrel, -) was designed (see Figure 1). Initially slower velocities were desired. As the project matured, the need for higher velocities became apparent. Three different gun barrels designs were necessary to cover the velocity range. Although all three are very similar, they differ in bore diameter, cartridge size, and barrel material. The internal barrel length was held constant at 6.75 -in.

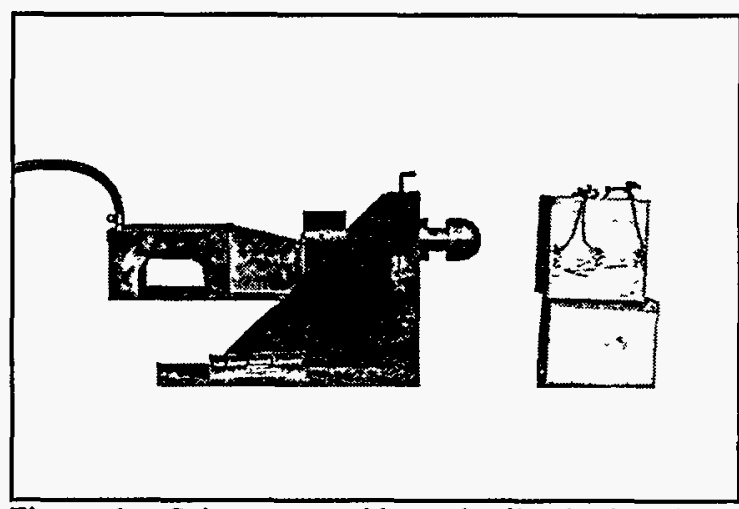

Figure 1: Spigot gun with projectile in barrel and photodiode velocity screen box.

The first barrel design with a 0.5 -in. diameter bore and 2.0-in. outer diameter (OD) used a standard .45-70 rifle cartridge to contain the primer and powder. The barrel screwed into the 4 inch OD breech with a course Acme thread. The muzzle was designed with 4 ports to vent the powder gasses and reduce projectile tip-off at exit. The projectile had a 0.5 -in. diameter spigot (shank), 6.25-in. long with a 3.0-in. diameter hemispherical head, and used a 0.5-in. long polyethlyene obturator to seal the powder gasses. This combination worked well at low velocities (as low as $13.7 \mathrm{~m} / \mathrm{s}$ ) but the spigot buckled in the barrel at higher velocities. Hence, a new gun barrel was designed.

The second gun barrel required a simple modification of the first design, and changes in the spigot and obturator. The bore was increased to 0.75 in. diameter, the spigot was shortened to 5.25 -in, and the obturator was lengthened to 1.5inch. All other aspects of the design remained the same. We have completed a total of 148 shots with velocities ranging from 24.5 to $94.6 \mathrm{~m} / \mathrm{s}$. Two problems were encountered at high velocities. At velocities greater than $64.0 \mathrm{~m} / \mathrm{s}$ the higher pressures expanded the base of the mild steel spigot causing excessive friction in the barrel. This problem was reduced by tapering the end of the shank by 0.01 -in., allowing velocities up to $\sim 76.2 \mathrm{~m} / \mathrm{s}$. Even higher velocities required shanks of A-1 tool steel hardened to Rc-51. At velocities greater than $82.3 \mathrm{~m} / \mathrm{s}$ the pressures were high enough to cause low cycle fatigue with attendant barrel ruptures. Thus, a third gun barrel design was required.

The third gun uses a barrel made of AISI 4340 steel heat treated to Rc-37. It has the same 0.75-in. diameter bore as the second gun but uses a .45 -Basic cartridge (the same as a $.45-70$ but one inch longer). The barrel OD was increased to 3 inches and the receiver OD was increased to 6 inches. The projectile and obturator design remained the same as for the second gun. A total of 43 shots have been fired with this design at velocities between 73.5 and $109.5 \mathrm{~m} / \mathrm{s}$ with no problems.

The combined powder curves for the second and third guns are shown in Figure 2. As with any powder gun, certain loads produce more velocity scatter than others. The two guns do not follow the same curve as a result of the different cartridges used.

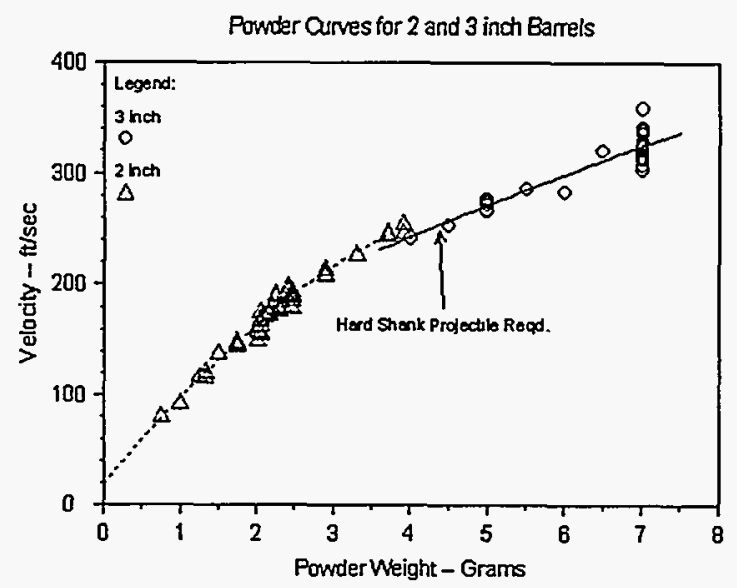

Figure 2: Powder curves for 2.0-in. and 3.0-in. OD barrels.

Because of the projectile mass the powder burns almost as if it was in a closed container. As such the burn characteristics do not match any published data and typical computer predictions are of little value. In our initial attempts to measure the burn pressure using 100,000 psi piezoelectric pressure transducerswe consistently overranged them and had on several occasions literally blown them out the side of the barrel. Subsequently we instrumented the barrels with 
both hoop and axial strain gages and have measured pressures as high as $161,000 \mathrm{psi}$ in the chamber. An example of the strain measurements are shown in Figure 3 and the computed pressure is shown in Figure 4.

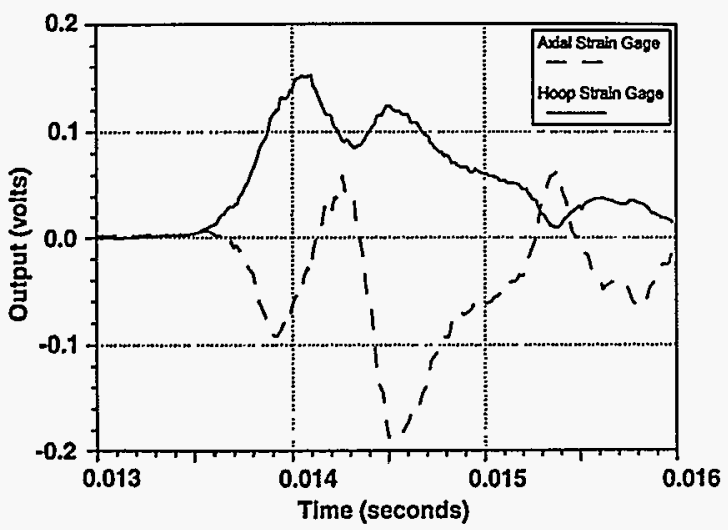

Figure 3: Hoop and axial strain measurements obtained from the high pressure barrel design.

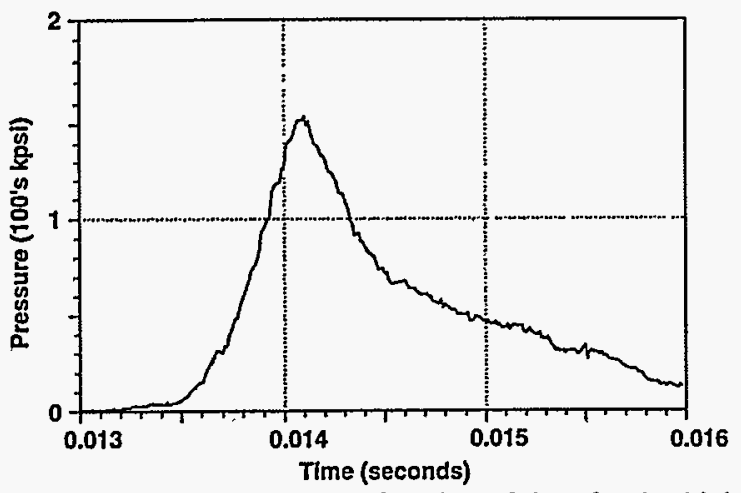

Figure 4: Bore pressure as a function of time for the high pressure barrel design.

A gun barrel is neither a closed cylinder nor an open one. One might expect the axial strain measurement to lie between these two conditions, but as can be seen in Figure 3, this is clearly not the case. The strain starts out negative (compressive) and then oscillates at high amplitude. The initial compression is due to the Poisson effectcombined with the undefined end conditions of the barrel. The initial contractions of the material then excite the axial natural frequencies of the barrel. This explanation was verified by comparing an FFT analysis of the data with a finite element analysis of the gun. The frequencies present in the data match the computed axial natural frequencies of the gun assembly.

Although the distance from the gun to the target is only 4 feet, the extremely violent recoil of the gun requires a massive sliding gun mount to provide reproducable hit points. The hit point is a function of powder load, but is quite consistent for a given load. Tests are performed with plywood (inert) targets to verify both velocity and hit point prior to each live target test.

For safety reasons, arming energy is not permitted on the firing mound until all personnel are removed. An electrically actuated, pneumatically driven firing mechanism was designed for this purpose. Final electrical and compressed air connections are made after the area is cleared of all personnel. Further details of the gun design, the powder loads, and the velocities may be found in the Los Alamos National Laboratory (LANL) report $(1)$ on the first series of experiments.

\section{PBX 9501 SAMPLE/SPECIMEN HISTORY}

Four different lots of PBX 9501, produced by Holston to PBX 9501 specifications defined by LANL, were used in the various target designs. The lot numbers, average densities, and dimensions are given in Table 1. They differ in age, density, and in formulation. The oldest lot, 685-002, was formulated with the diphenylamine (DPA) stabilizer and the other three with Irganox.

Lot 731-003, the youngest lot, was first tested in the large, thick targets. Lot 730-010, formulated in 1989, still meets the PBX 9501 molding powder specifications, and was defined as the 'baseline' lot for the small, thin and thick targets. These specimens were machined to dimension from samples pressed in 1997 and 1998.

Specimens from lots 685-002 and 730-005, formulated in 1977 and 1981 respectively, were defined as the 'aged' specimens. They differed from the baseline material in age of the pressed samples, and in density, with the aged material being slightly denser. These 'aged' specimens were machined from pressed samples, 182 and 142 months old respectively. These specimens were only used in the small, thin targets.

Table 1: PBX 9501 lot numbers, average density, and specimen dimensions.

\begin{tabular}{ccc}
\hline PBX 9501 Lot \# & $\begin{array}{c}\text { Average Immersion Density of } \\
\text { Specimens }\left(\mathrm{g} / \mathrm{cm}^{3}\right)\end{array}$ & Specimen Dimensions (target design) \\
\hline $685-002$ & 1.840 & 5.00 -in. diameter by 0.50 -in. thick (small, thin) \\
$730-005$ & 1.840 & 5.00 -in. diameter by 0.50 -in. thick (small, thin) \\
$730-010$ & 1.830 & 5.00 -in. diameter by 0.50 -in. thick (small, thin) \\
& & 5.00 -in. diameter by 1.00 -in. thick (small, thick) \\
$731-003$ & 1.836 & 5.75 -in. diameter by 1.00 -in. thick (large, thick) \\
\hline
\end{tabular}




\section{TARGET DESIGNS}

The following target descriptions are based on the chronological design evolution with respect to material and diagnostic requirements and availability.

The first test series of ten targets was based on the original large, thick target design. The basic design started as a modification of the target assembly used by Chidester et al. ${ }^{(2-4)}$ with subsequent changes for different diagnostics, including pinducers, a carbon film gauge, and a thermocouple. This basic target design, depicted in Figure 5, consisted of a cylindrical holder with a 0.5 -in. thick back plate, $\sim 0.125$-in. thick disc (cover plate), and retaining ring machined from 304ss. The targets were assembled with seven evenly spaced bolts, and the eighth bolt was used to secure the assembly to a mild steel backing plate as described below. The 1997 LANL report ${ }^{(1)}$ provides a more detailed description of the basic target design, dimensions, and modifications.

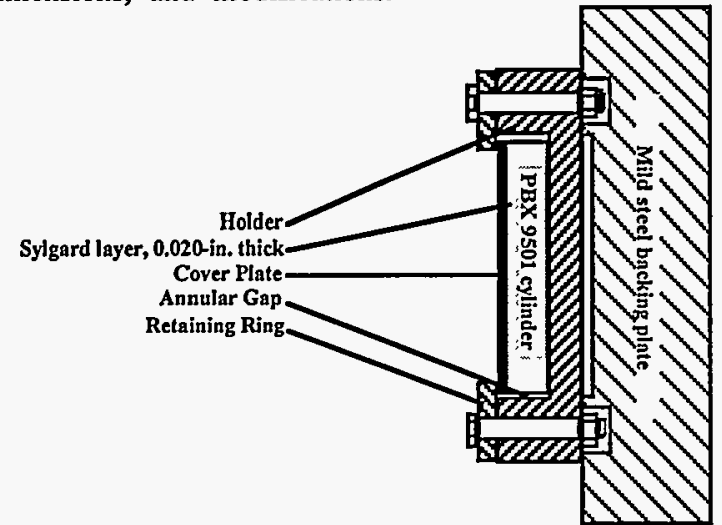

Figure 5: Basic target design used for the modified Steven tests.

The PBX 9501 used for the large, thick targets, was isostatically pressed, and machined to dimension. Immersion densities were determined for each of the PBX 9501 articles, 5.75-in. diameter by 1.0-in. thick, with an average density of $1.836 \mathrm{~g} / \mathrm{cm}^{3}$. The $\mathrm{HE}$ cylinder was centered in the target holder and slightly compressed by the target design. A Sylgard 184 layer, $\sim 0.020$-in. thick, was placed between the $\mathrm{HE}$ and the cover plate. The original design dimensions allowed for an annular gap of 0.125 -in. between the HE O.D. and the holder I.D.

The mild steel backing plate, 12.0 -in. square by 3.0 in. thick, served as a high impedance backing plate for the target assembly. For the first test series of ten targets, a 0.25-in. deep, -6.0 -in. diameter circle was milled into the center of the steel backing plate to allow for rear surface deformation of the target. The depth was increased later to 0.75 -in. for the small, thick and thin targets.

Subsequent, large, thick target designs were changed in several ways to minimize the material and machining costs and to allow for other diagnostic measurements. These included changing the cylindrical holder and retaining ring materials to mild steel, and the back plate thickness was increased to 1.0 -in. thick. Two of the several different designs will be described briefly here: the 'Glass-backed', and 'Not Half Bad (NHB)' designs.

The Glass-backed target design employed two 5.0in. diameter by 1.25-in. thick windows stacked together, and assembled directly behind the HE surface. The intent was to use a fast framerotating mirror camera to record the crack/fracture evolution of the $\mathrm{HE}$ after impact, and to witness any evidence of ignition light. Four targets have been machined for this purpose.

The NHB design employed a sandwiched array of film gauges behind the $\mathrm{HE}$ between the thin $(0.125$-in.) and thick (1.0-in.) back plates. The target holder was modified into three different cylindrical layers for this purpose: the retaining wall, the thin and the thick back plates. Four targets of this type have been tested.

The small, thin targets were designed with $\mathrm{HE}$ and target material constraints in mind. For this purpose the $\mathrm{HE}$ diameter and thickness were decreased to 5.0-in. and 0.5-in. respectively. Accordingly, the A-36 steel target holder, and retaining ring were manufactured with smaller diameters to retain the original annular gap width. The back plate thickness was changed to 0.75-in. thick, and stock, 0.118-in.-thick, 304ss material was used for the cover plate to reduce machining time. The small, thick targets differed from the small thin targets in the thickness of the PBX 9501 article, and the holder depth.

\section{DIAGNOSTICS}

Different diagnostics have been used to evaluate the velocity, timing, pressure, strain profiles, and level of reactivity of the mechanical loading event. These diagnostics included, but were not limited to, $\sim 50 \Omega$ carbon and manganin film gauges, $\sim 50 \Omega$ pinducers, PVDF and LANL-designed impact foil switches, strain gauges, and piezoelectric pressure sensors. A short description will be provided on the various devices used and their purpose.

\section{Projectile Velocity}

One of the most important performance characteristics of the gun is the projectile velocity. 
Because the powder gasses are vented before the spigot completely exits the muzzle, a velocity measurement can be obtained very close to the muzzle. A cheap, easily replaceable light box/photodiode system was designed for determining the projectile velocity. It is composed of three sets of halogen lights and photodiodes spaced at three inch intervals in a wooden frame. The data records yield three profiles which can be used as two independent velocity measurements. An average velocity is then determined from these two values.

\section{Impact Timing}

The impact timing measurement requires a durable diagnostic with a reliable trigger at these slow projectile velocities. Triggering performance was verified by projectile impact on the inert targets. Our endeavors have resulted in an evolution of different diagnostics. The first was a double wire grid separated by 0.5 -in. This proved to be late in response time due to stretching. The second method used a copper impact foil switch, which again proved to be unreliable. Next, we tried a three path parallel light grid using halogens light sources and photodiode. However, the supporting electronics were not adequate to provide reliable triggering. The subsequent modification used built-in amplifiers with the three path configuration. This system produced large sharp trigger signals; however, it occasionally produced significantly premature triggers.

In the interim, a temporary solution was developed with three parallel graphite rods. The rods were connected in series with resistors to produce a total resistance of $\sim 50 \Omega$. A voltage was applied to the circuit, and the three rods were monitored for the first arrival of a high or low pulse as a result of projectile impact. This method still produced some jitter in the trigger for the last experiments and more in the first two instrumented tests. Hence a methodology was required to establish a common time base for the data associated with these tests. This methodology will be described in more detail in the data analysis.

Another iteration on the impact triggering produced a laser switch comprised of a laser with 12 passes between mirrors with a photodiode detector. This method was prone to pretiggers similar to the halogen lamp-photodiode array. The current method employs a Dynasen PVDF gauge to record the time of impact. The gauges have proven be reliable, but are quite noisy and can cause interference on subsequent diagnostic records.

\section{Impact Location}

A grid of 14 to 18 foil switches, spaced with centers separated by 0.5 -in., were used in the first large target series to determine the impact location. The projectile surface served to complete an open circuit on each foil gauge. The impact location was determined by using a record of the trigger times and triangulation.

This method also suffered from premature and late time triggering. Hence, it was only used on a limited number of targets. Thus, in the absence of this diagnostic technique, the impact locations were determined from the remaining cover plate and/or holder evidence.

\section{Target Diagnostics}

The target diagnostics were changed from design to-design. This section will describe the type of diagnostic used, the location of the diagnostic in the target design, and the purpose of the diagnostic.

Dynasen $\sim 50 \Omega$ carbon film gauges, with the element centered on the front surface of the PBX 9501 and potted in the Sylgard 184 layer, were used on the last six large, thick targets. Their purpose was to measure the low pressure produced in the $\mathrm{HE}$ at the time of impact and any subsequent reaction. Recent measurements have shown that the carbon film gauges exhibit sensitivity to the different light levels produced by detonating PBX 9501. Feasibility tests are being performed to assess the possibility of using the carbon film gauges as a diagnostic to identify ignition locations. However, the results summarized here have not been reanalyzed for this possible contribution.

Low pressure, 1 to $16 \mathrm{kbar}$, and timing profiles were also recorded with Dynasen $\sim 50 \Omega$ pinducers. A detailed description and calibration procedure for these devices was given by Lucht and Charest. (5) The pinducers were used in both radial and back surface positions. Further details of the mounting procedure, and location in the target designs have been reported previously. (1)

A fast response, chromel-alumel thermocouple, calibrated to $1400^{\circ} \mathrm{C}$ was also used on a few of the large, thick target designs to measure the temperature of the event during impact. However the thermocouples did not yield much useful information because the measured voltages correlated to temperatures much higher than that if the calibration is extrapolated. It is likely that the flow of HE along the back plate of the target quickly removed the chromel-alumel junction, and the remainer would have behaved basically like an ionization pin. If this scenario is correct, then the initial fast rise of the temperature may be correct, but beyond $1400{ }^{\circ} \mathrm{C}$ we are probably observing regions of hot, ionized, reacting material flow.

The four NHB target designs employed radial arrangements of manganin and/or carbon film gauges sandwiched behind the PBX 9501 in a layer between 0.125-in. thick, and 1.0-in. thick steel. The gauge elements were located at four different radii, $0.5,1.0$, 2.0 , and 2.5-in., and aligned relative to predicted crack patterns in circumferential and radial directions. 
The back plate of many of the live targets have been instrumented with two strain gages mounted at right angles to each other to measure back plate deformation. Several iterations of gage and leadwire mounting systems were tried to increase data collection time. Currently, the gages and lead wires survive throughout the initiation of the violent reaction and for some time thereafter. Data loss occurs when the gages are either crushed between the back plate and the rear mounting block or when cables are destroyed by the target dissassembly.

\section{Violence of Reaction}

Knowledge of the degree of violence produced by the mechanical loading of the PBX 9501 is desirable for probability risk assessments. Our initial experimental efforts relied solely on the remaining physical test evidence to estimate the extent of reaction relative to a PBX 9501 steady-state detonation. For this purpose a thick, large modified Steven target was intentionally detonated with a detonator and Detasheet booster to evaluate the level of damage to the target materials and PBX 9501. It is important to note that none of the large, thick target designs have demonstrated the same degree of damage to the target materials. However, in many of these tests the PBX 9501 was completely consumed by the reaction and ensuing events.

The subsequent experimental series were enhanced with the additions of a ballistic pendulum and blast overpressure gauges to evaluate the violence of reaction. The two entirely different methods produce strikingly similar results not only for a high level reaction but for very low levels of reaction as well. A short description of each follows.

\section{Ballistic Pendulum}

A classic ballistic pendulum was designed to measure the violence of reaction relative to a PBX 9501 steadystate detonation. The pendulum is shown in Figure 6 . The pendulum weight can be varied by adding or removing 95 lbs plates from the pendulum box. Only two weights have been used for the data reported here. The pendulum weight was adjusted to $2098 \mathrm{lbs}$ and 3198 lbs for the targets using 0.5-in. and 1.0-in. thick explosive respectively. The mild steel backing plate added an additional $\sim 110$ lbs. to the overall weight. Momentum transfer calculations were performed for varying amounts of detonating explosive for the two pendulum weights. These were benchmarked with four PBX 9501 calibration charges. The degree of pendulum displacement is measured using two independent passive measurements. One is a friction pivot-arm that is displaced by the pendulum, which locks in place at the peak of the swing. The other is a light cable, labeled with a marker, anchored to the pendulum box over a pulley mechanism. The cable is pulledby the swing of the pendulum, and the marker displacement is measured.

Ballistic Pendulum Calibration Tests 18 July 1997

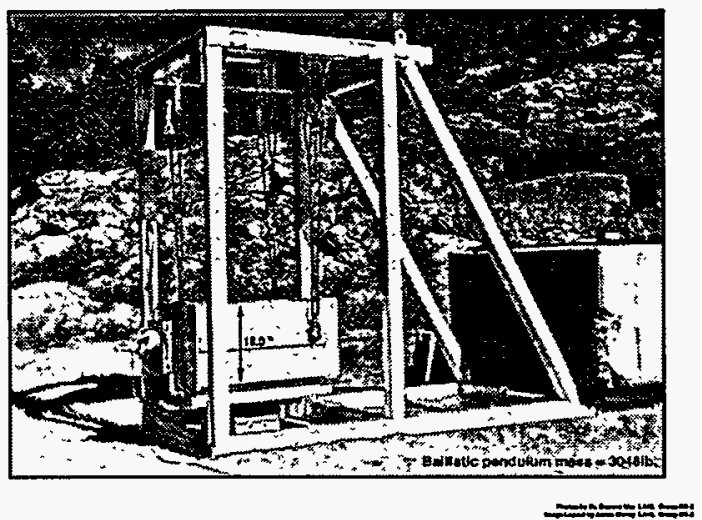

Figure 6: Image of ballistic pendulum with one of the PBX 9501 calibration targets attached.

The blast overpressure gauge measurements were recorded with two 1000 psi maximum PCB, Piezotronics Inc. Model 102A15 ICP High Frequency transducers. These were used in face-on mode positioned at $45^{\circ}$ off of the projectile axis at a distance of $\sim 10 \mathrm{ft}$. from the target face. Pressure and timing profile calibration occurred simultaneously with the ballistic pendulum calibration. The blast gauge measurements were averaged with the passive data obtained from the ballistic pendulum and reported as the average energy release.

\section{Delay to Reaction}

The delay to the violent reaction resulting in the destruction of the target was estimated by several different methods. For targets equipped with the impact foils, pinducers, strain gages, carbon film gauges, and/or manganin gauges, the time was interpolated from the data record indicating lead destruction. A comparison of the timing between the projectile impact and the arrival of the blast wave at the blast gauge also provided a qualitative time base for the delay.

\section{POST-TEST TARGET CHARACTERIZATION}


The post-test characterization methods can be categorized into non-intrusive and intrusive testing to examine the damaged state of the target materials and the PBX 9501. A brief summary of the techniques will only be provided here. Several of these techniques and their results will be described in more detail in a future paper by Phillips, et al.(6)

Nonintrusive techniques included the front surface dent and rear surface deformations evaluation, visual examination of the front surface of the PBX 9501, X-rays of the cracked PBX 9501, and ferrite scope measurements of the damaged target materials. The dent and deformation information was used to calibrate and verify the materials models being used in the finite element DYNA2D calculations. The visual examination of the top surface 1) verified that the PBX 9501 had yielded to the impact and expanded to fill the annular gap, and 2) displayed distinct types of crack structures, circumferential (C), radial (R), and mixed (M) (see Figure 7), with evidence of shear dislocation along some of the cracks. The static $x$-rays show evidence of the crack depths and widths relative to each other.

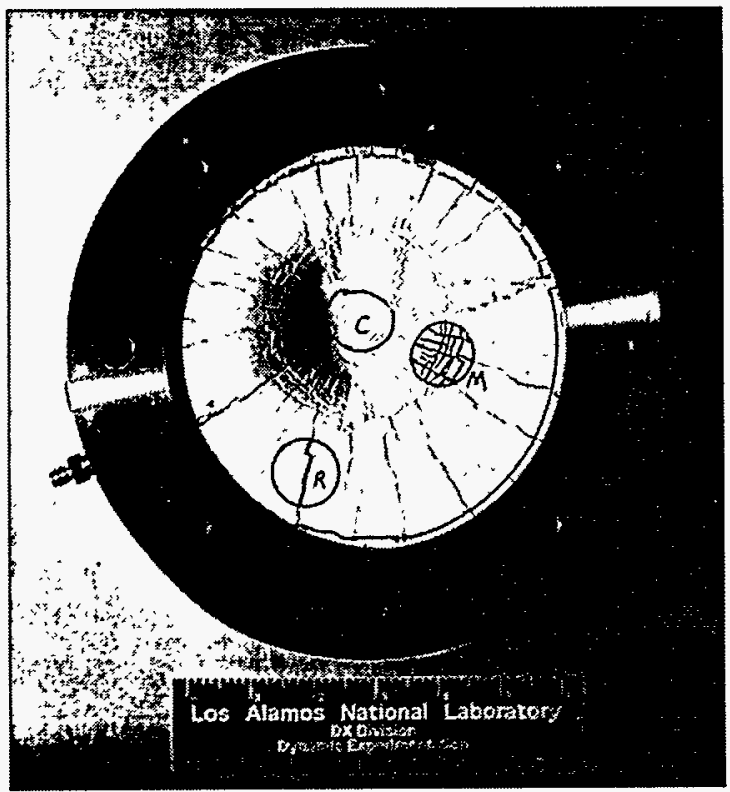

Figure 7: Image of large, thick, target K3-1278 showing the crack structure and damage to the PBX 9501 after a $72.2 \mathrm{~m} / \mathrm{s}$ impact.

Fisher ferrite scope measurements on target remnants from an intentionally detonated target were compared with those from a violent reaction to look for quantitative differences in the amount of shearing experienced by the target materials. The material shearing is believed to result in the conversion of the austenitic phase of the 304 stainless steel to the martensitic phase.

The intrusive techniques included sampling for immersion density, polarized light microscopy (PLM), and scanning electron microscopy (SEM) measurements. Core samples from the three different crack regions, were taken from 3 of the large, thick targets for the immersion density measurements. These data display a trend from lower density directly under impact to higher density near the target edges. This trend is also supported by the static x-ray data of the impacted targets, which display a lower density immediately under the impact point. PBX 9501 specimens recovered from both quenched and reacted targets were examined by PLM and SEM techniques. Some show evidence of twinning, melt and/or decomposition.

Post-test characterization is still ongoing, and additional non-intrusive and intrusive techniques are being investigated to further characterize the damaged state of the PBX 9501. These results will be reported in future publications. $(6,7)$ These include probing the surface of the PBX 9501 with a second harmonic generation technique $(8)$ to look for polymorphic changes in the HMX as a result of the mechanical loading. The existence of a different polymorph will be further verified with FTIR and powder $\mathrm{x}$-ray diffraction of small PBX 9501 specimens. Changes in the thermal behavior of the PBX 9501 will also be investigated with modulated Differential Scanning Calorimetry (DSC).

\section{MODELING}

Calculations using DYNA2D, SPRONTO and DYNA3D have been performed to support these, and other, low amplitude insult experiments. These are described in more detail by Scammon, et al.(9) The DYNA2D finite element calculations employ an ignition criterion as a function of a characteristic constant, pressure, time and the maximum shear strain rate to evaluate the threshold level to reaction. The SPRONTO code is a result of embedding the LANL statistical crack mechanics model, SCRAM, into the Sandia mechanical deformation code PRONTO. It uses solutions of a one-dimensional heat equation, perpendicular to the planes of penny-shaped cracks excited into two-dimensional grinding motion by the impact, to compute the chemical response of the PBX 9501. These calculations allow us to study pressure and strain rate variables, to investigate structural aspects of the experiment, and to predict velocities required for reaction. Structural analyses have played an active role in this project beginning with the original target design and continuing through analyses of the experimental results. Alternative designs and various ideas for active instrumentation 
were examined as part of the experiment evolution process. Predictions of reaction are used to guide these design studies, even though we do not yet have enough experimental data to fully calibrate any of the models.

\section{RESULTS AND DISCUSSION}

The diagnostic evidence from the large, thick target series of tests has been previously reported in detail. ${ }^{(1)}$ Nonetheless the results will be summarized here for comparison with the other series of tests.

In the large target series, the low amplitude mechanical loading of lightly confined PBX 9501 produced violent reactions well below that required for shock-to-detonation transition (SDT). Even though the violence of reaction was not quantitatively measured in this series, the pressure gauge data coupled with the post-test target fragments indicate a significant energy release sufficient to cause severe damage to the target but unequal to a classic $\mathrm{CJ}$ detonation. Target damage included hemispherical deformation and shearing of the cover plate, fragmentation of the cover ring, and material failure in the steel holder. Significant burn and scorch marks were also evident on the holder and cover plate interior surfaces. Ferrite scope measurements of target fragments suggest a higher degree of shear induced across the back plate of the mechanically impacted targets versus the intentionally detonated target. It is also evident that impact generated compression, fracture, and shear flow produce significant heat with very little delay in quenched and damaged targets. At velocities above threshold this is subsequently followed by a delay, from 0.5 to $1.0 \mathrm{~ms}$ long, for the reaction to escalate to a violent climax.

With the exception of one test, all of the targets that reacted violently resulted in complete consumption of the PBX 9501. PBX 9501 retrieved from the semiviolent event and the quenched targets were retained for post-test characterization. PLM and SEM evidence of the PBX 9501 retrieved from the semi-violent event show increased evidence of twinning in the HMX crystals, most likely due to high levels of stress induced by impact, and local regions of melt and gas pockets suggesting possible decomposition. SEM images of PBX 9501 specimens taken from a quenched target also show evidence of crystal twinning in HMX in the different crack structures with some intragranular cracking apparent.

Compared to the typical small scale Taylor test, this particular test design shows remarkable consistency and a very sharp velocity threshold between 72 and $75 \mathrm{~m} / \mathrm{s}$ (see Figure 8). Although the data are noisy, they show a consistent progression of reaction severity with a corresponding decrease in the reaction time as the impact velocity is increased.

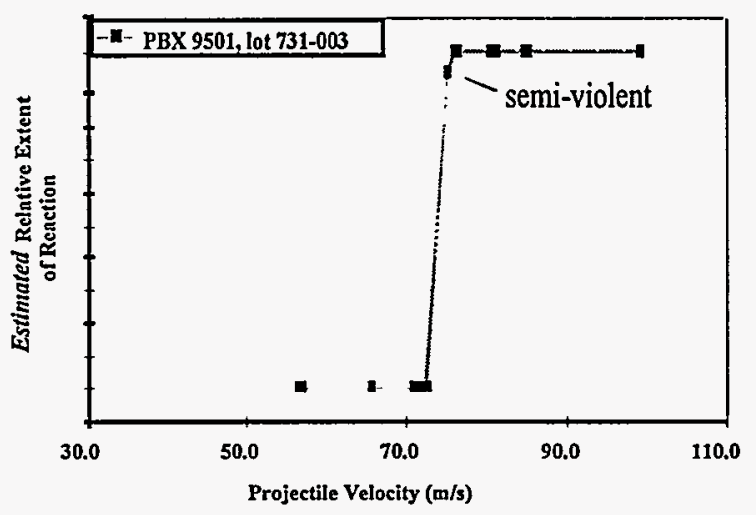

Figure 8: Threshold to reaction as a function of velocity for the large, thick Steven targets.

Although the gauge data from this target series are by no means conclusive, they indicate that heating and possibly burning starts over a large area and may sensitize a large portion of the volume of damaged HE. During the time between the impact and the violent climax, at least one reactive wave is generated and moves across much of the mass of material at velocities ranging from 1 to $2 \mathrm{~mm} / \mu \mathrm{s}$. Thus, the wave is probably locally subsonic and does not have a well-defined front.

Only two each of the Glass-backed targets have been tested. We encountered lighting and camera difficulties with these tests. Nonetheless, the preliminary analysis shows a significant shift upward in the threshold velocity, between 96 and $99 \mathrm{~m} / \mathrm{s}$, required for violent reaction to occur. It is assumed that the lower yield strength for the glass versus steel allows the target to relieve the pressure produced by the reaction more readily and effectively quench a reaction much quicker. Hence, higher velocities would be required to achieve the same pressure conditions required to sustain a reaction as in the steel-backed targets. Further tests are required to examine the merits of the glass-backed target configurations.

Of the four NHB target tests completed, the fourth target, $\mathrm{K} 8-2542$, impacted at $103.1 \mathrm{~m} / \mathrm{s}$,provided the most considerable insight into the level of reactivity of the larger targets and as to the timing of reactive events within the explosive package. This was the first large target tested with the combination of the ballistic pendulum and blast gauges, which measured an average energy release of $67 \pm 4 \%$. Based on the comparable level of target damage and expenditure of PBX 9501, we assume that this energy release is similar in violence to the most reactive large target previously tested.

Sixteen manganin gauges were used in the sandwich layer.for Target K8-2542. Figure 9 shows the pressure profiles for all of the gauge outputs. Signal first arrival varies considerably from location to location. Figure 10 is a contour plot of the first-arrival at the NHB 
gauge plane. It clearly defines the hit location on the target as being slightly high and right. This agrees very well with the recovered portion of the target cover plate. Expansion of these data shows later common events within the explosive and can be used to deduce relative timing and position of ignition and burn. The data strongly imply that the ignition point was directly under the point of impact and near the front cover in this target configuration.

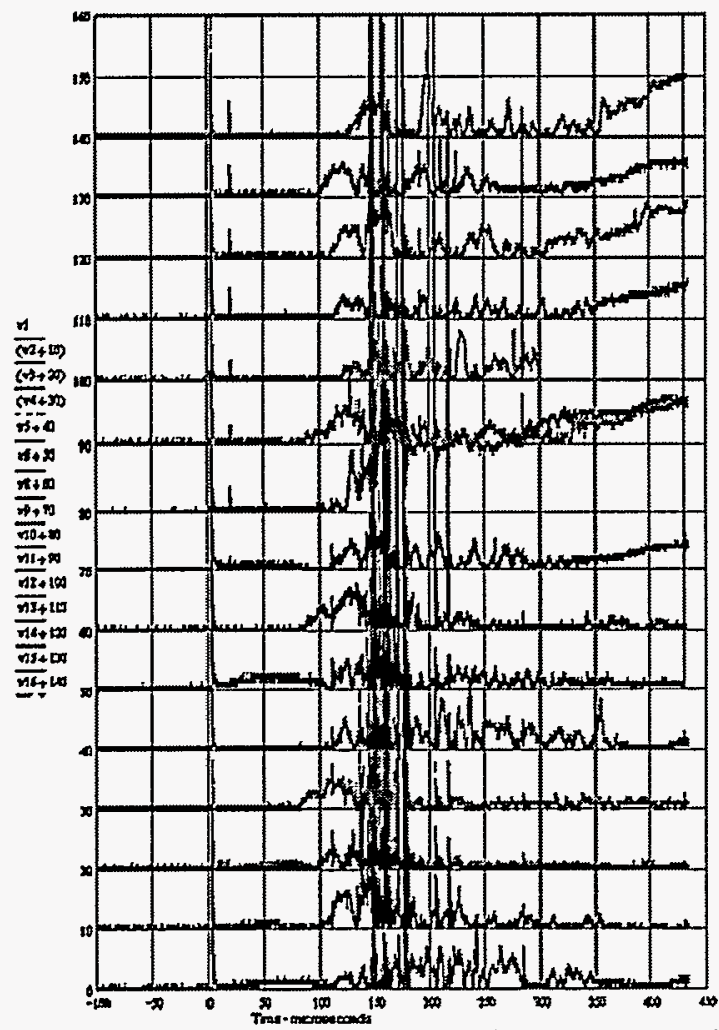

Figure 9: Pressure profiles for 15 of the 16 manganin gauges used in test K8-2542. Gauge 7 failed to record any data.

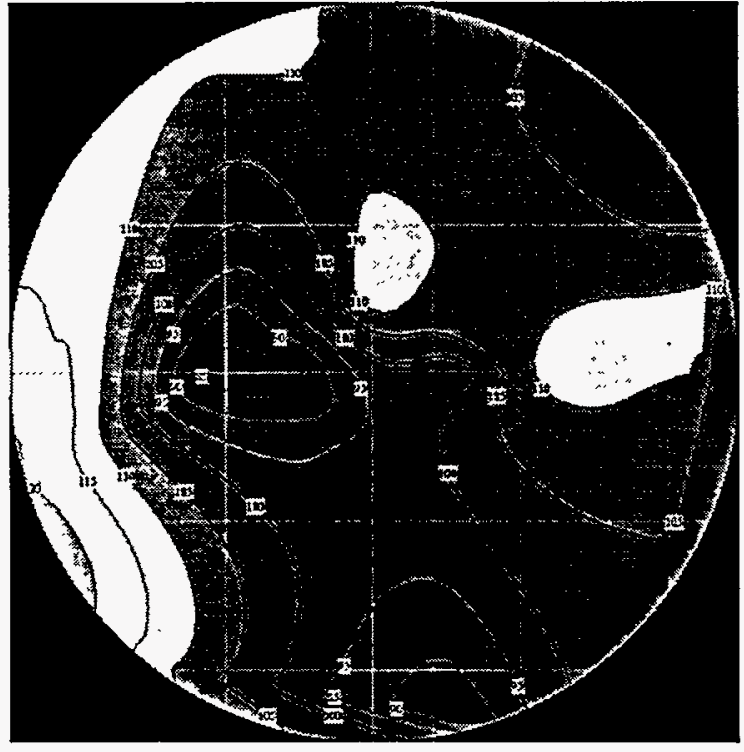

Figure 10: Contour plot of first arrival times for the manganin gauge records from test K8-2542.

Less target damage was produced by the reaction in the small, thin targets resulting in deformation to the retaining ring and cover plate, and little or no deformation to the holder. Similar evidence of burning or scorching as seen in the large, thick targets was not seen with the smaller target tests. It is possible that the lower level of damage can be directly attributed to the smaller quantity of PBX 9501 that reacts. This assumption is further supported by the average energy release, which never exceeded $41 \%$ for this target size. PBX 9501 fragments were recovered from each of the reactive events, decreasing in quantity as the projectile velocity was increased.

Analogous to the large target series, the threshold behavior for the small targets, both thick and thin demonstrate the same sharp consistency without crossovers. However, the most notable difference can also be seen in the threshold shift to lower velocities (See Figure 11), ranging from 54.4 to $55.9 \mathrm{~m} / \mathrm{s}$ for the ten thin PBX 9501 lot 730-010 targets and from 51.8 To $53.0 \mathrm{~m} / \mathrm{s}$ for the eight thin, aged PBX 9501 targets. The slight difference in threshold values between the lot 730-010 targets and the aged PBX 9501 is postulated to be due to velocity measurement errors, assuming $2.3 \%$, and to the differences in densities. The effect of density on mechanical loading events is currently being explored further with lab-scale, quasi-two dimensional target testing. $(10)$ The preliminary analyses suggests that higher density PBX 9501 experiences more shear strain under mechanical loading as compared to a lower density piece. This result suggests that if shear strain plays a dominant role in the ignition sequence of events, we would expect a lower threshold to reactivity for the higher density material. 
Further tests are required to verify the relative quantity of shear strain and it's role in the ignition behavior.

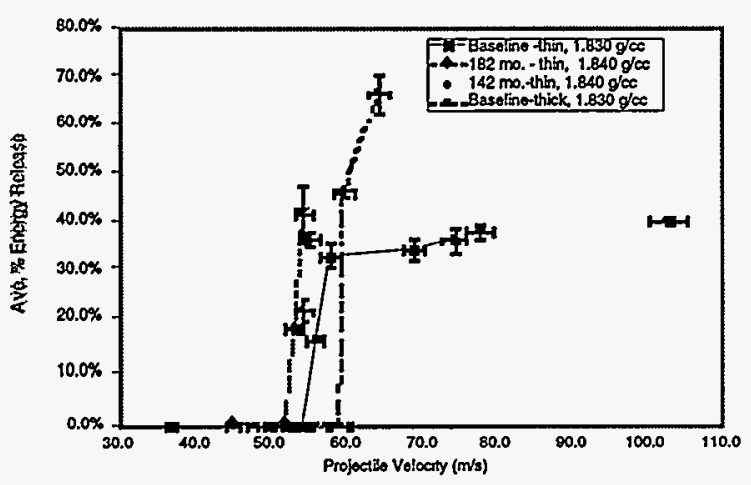

Figure 11: Threshold to reaction for the small, thick and thin Steven targets. The PBX 9501 lot 730-010 is referred to as the baseline lot.

Thicker, small targets were tested to determine if the $\sim 20+\mathrm{m} / \mathrm{s}$ difference between the threshold ranges was, in part, due to the thickness of the PBX 9501 impacted. Five targets with 1.0-in. thick PBX 9501, lot 730-010, were tested for this purpose. Three of the targets quenched, and two resulted in violent reaction. The degree of target damage to the cover, retaining ring, and holder resembled the damage witnessed with the large, thick target designs with the average energy release increasing to $67 \pm 4 \%$. These results support the concept that in increase in the HE mass leads to more confinement damage; however, there was only a slight change in the threshold velocity range from 59.1 to $59.6 \mathrm{~m} / \mathrm{s}$. Hence the increased threshold velocity is most likely due to different factors other than the thickness of the HE.

An examination of the physical target differences and similarities and gauge records produces additional insights regarding this behavior. Back plate strain gages were used on all of the small targets.

The strain gage records show a strong consistency in the impact induced reaction sequence. The records from the thick, small target tests K8-2480 and K8-2484 are shown in Figure 12. The explosive initially behaves as an elastic solid, transmitting force to the back plate. At about 2000 microstrain on the back plate, the explosive starts to crush and flow radially. The force on the back plate levels off and then starts to decrease until the annular gapbecomes filled and the pressure on the crushed explosive begins to build again. If the impact velocity is high and long enough, as in test K8-2480, to generate sufficient reaction in the explosive, the pressure is sustained and will continue to build until a violent reaction occurs. If the velocity is low, as in $\mathrm{K} 8$ 2484 , insufficient reaction occurs to sustain the pressure. Consequently the reaction is quenched and the projectile simply rebounds, releasing the force on the back plate. Because these strain gage records clearly show the mechanical behavior and reaction timing of the explosive, they become excellent data for benchmarking the codes. As expected, the delay to violent reaction from impact decreases as the projectile velocity is increased. At significantly high velocities the strain records show that the reactive event occurs even before the force on the back plate begins to decrease.

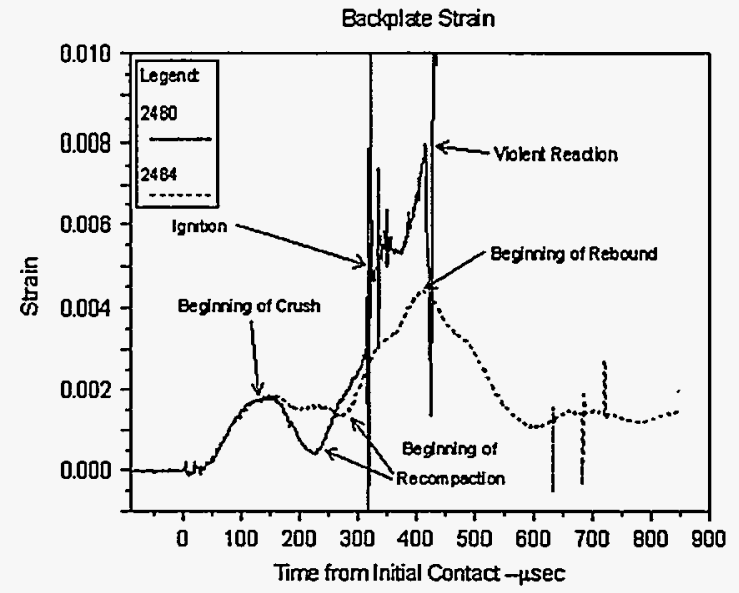

Figure 12: Strain gage records from tests K8-2480 and K8-2484. Test K8-2480 resulted in a violent reaction, and test $\mathrm{K} 8-2484$ was quenched.

Semi-quantitative delays in reaction times can also be determined comparing the blast gauge time profiles to impact time relative to a prompt detonation of the PBX 9501. This analysis of the small, thin targets produce delay times decreasing from $\sim 2.8$ to $1.3 \mathrm{~ms}$ as the projectile velocity is increased to $\sim 77 \mathrm{~m} / \mathrm{s}$. Notably the delays to reactions in the small, thick targets were shorter, ranging from $\sim 0.2$ to $0.9 \mathrm{~ms}$ at velocities of 64.3 and $59.6 \mathrm{~m} / \mathrm{s}$ respectively. The magnitude of these delay times correlates well with those evaluated for the large, thick targets, which ranged from 0.5 to $1.0 \mathrm{~ms}$ long. It is probable that the delay times to reaction are shorter for the thicker targets because a larger HE mass is affected by the impact and the ensuing reaction. This results in faster increase to the critical pressure required to achieve the violent reaction.

Interestingly, the threshold velocity for the small, thin targets could be predicted to within $\pm 3 \mathrm{~m} / \mathrm{s}$ with prior knowledge of the dent information from quenched targets. A linear regression fit to the projectile velocity as a function of the measured dent depths allows one to extrapolate to a velocity where the PBX 9501 thickness will be fully 'pinched.' For the small, thin targets, tested with the 730-010 PBX 9501 lot this corresponded to a velocity of $55 \pm 3 \mathrm{~m} / \mathrm{s}$ to pinch the 0.5-in. thickness. 
A similar analogy for the thicker targets, both large and small, does not exist based on the remaining physical target evidence. In fact, this type of fit would overpredict the threshold velocity range. Thus, it is evident that a complete 'pinch' of the HE at the back surface is not necessarily the requisite precursor to a violent reaction. Hence, the question - what are the differences between the thick and thin targets ignition mechanisms, if any?

Perhaps a critical mass/density is achieved by the impact that is one of the necessary steps to the reaction sequence. Further examination of the dent data to determine a PBX 9501 volume displacement by the impact provides possible insight to this. Several assumptions are required for this analysis: 1) the impact is symmetrical, 2) the $100 \%$ theoretical maximum density (TMD) for PBX 9501 is $1.860 \mathrm{~g} / \mathrm{cm}^{3}, 3$ ) for densities less than $100 \% \mathrm{TMD}$, the free volume is attributed to porosity, 4) the available free volume in the target is represented by the sum of the annular gap volume and the porosity, 5) the energy deposited by the impact to the target is proportional to the velocity squared $\left(v^{2}\right)$ for projectiles of the same mass and diameter, 6) the data can be scaled by the HE length factor, 7) the steel used in the targets has essentially the same mechanical response to impact and material failure to violent reaction, and 8) the dent depth accounts for the combined mechanical response of the target materials and $\mathrm{HE}$. Using these assumptions we can calculate a factor, $k$, for each target as a function of the velocity, $\mathrm{v}$, ratio of displaced volume to free volume, $\left(V_{d} / V_{f}\right)$ and length scale, 1 , as follows:

$$
k=v^{2}\left(V_{d} / V_{f}\right) l
$$

A plot of these data as a function of velocity for the three different types of targets, ie. large, thick, and small, thick and thin is shown in Figure 13 for comparison. The $k$ factor was calculated with units of $\mathrm{m} / \mathrm{s}$ for velocity, and inches for length.

The data display a quadratic progression as a function of projectile impact velocity. A fit to the data produces the following equation for the threshold behavior of these particular targets and projectile design:

$$
y=2.12 x^{2}-140.1 x+2602.4
$$

Assuming this fit represents the threshold behavior for this particular target design and impactor, then we would expect that any data point below the curve would result in a quenched event, and any data point above the curve would result in a violent reaction. This relationship demonstrates a key point in the behavior between the displacement volume and the free volume available in the target. For those materials with higher densities the free volume will be slightly reduced, thus lowering the projectile velocity required to induce violent reaction. Analogously, to a larger degree, in targets without annular gaps, the free volume will be considerably reduced, and the threshold velocity would decrease correspondingly. The relationship also demonstrates why there should be no change in the threshold velocity with only a change in the HE thickness. However if the HE diameter changes, one would expect a change in the volume ratio, leading to a change in the threshold.

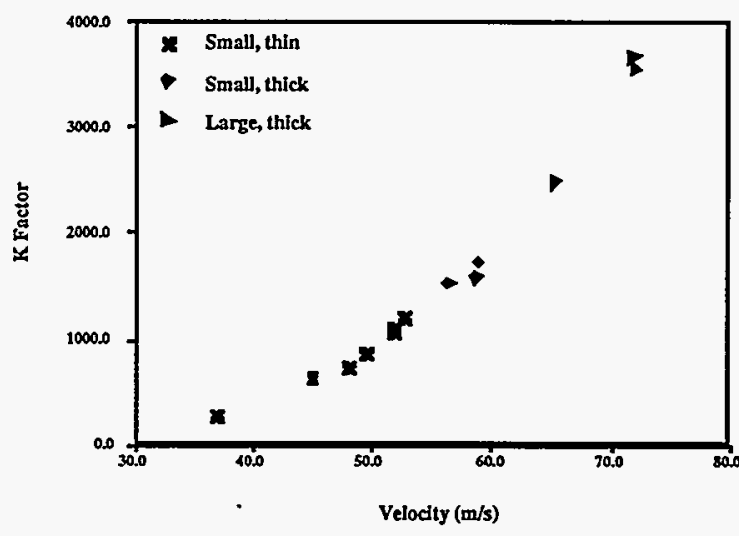

Figure 13: Plot of $\mathrm{k}$ factor as a function of projectile impact velocity for the modified Steven tests.

To extrapolate this relationship to other target and projectile designs would require additional scaling factors to account for differences, e.g. projectile mass, nose shape, target material yield strengths, and $\mathrm{HE}$ dimensions and $\mathrm{HE}$ mechanical properties behavior. Intuitively, we would assume that if the projectile shape was retained, and the mass decreased, that the threshold velocity would be increased. Changes in the projectile nose diameter would require prior knowledge of the change in the dent depth and corresponding displacement volume to determine the exact effect on the threshold curve.

\section{SUMMARY}

The mechanical loading behavior of PBX 9501 to a low velocity impact has been investigated using modified Steven target designs. Low velocity impact was produced by launching a $2 \mathrm{~kg}$. mild steel spigot projectile via a new powder driven gun design, from $\sim 20$ to $105 \mathrm{~m} / \mathrm{s}$, at the lightly confined, steel targets. The threshold velocity to reaction for various target designs, different PBX 9501 lots, and different high explosive (HE) thicknesses were evaluated and compared.

Various diagnostics were used to evaluate the pressure profile and timing, and target strain behavior relative to projectile impact. The pressure profiles and strain gage records provide evidence for a sequence of events as follows: low pressure event induced by 
impact, followed by $\mathrm{HE}$ material failure and recompaction which results in either violent reaction or a quenched event.

The violence of reaction, as measured by both passive and active techniques, is reported relative to a steady state detonation in PBX 9501. These results show that the violence of reaction is greater, and the delay to violent reaction is shorter with the thicker targets. However the violence was never equivalent to a steady-state detonation of the PBX 9501. The shorter delay times in the thicker targets are assumed to be a direct consequence of using a larger mass of $\mathrm{HE}$, providing more material for reaction.

Each type of target series has demonstrated a remarkable sharp threshold to reaction without evidence of cross-overs. The large and small target designs do lead to changes in the threshold range. However, if only the HE thickness is changed in the target design, little or no change is seen in the threshold velocity. The aged material tests resulted in a small shift down in the threshold. It is proposed that this is due to the slightly higher densities of these materials.

A threshold criterion curve was derived for these particular target designs and projectile characteristics based on the projectile velocity, displacement volume, target free volume, HE density and thickness. This curve suggests that there is a common mechanism to the

\section{REFERENCES}

(1)D. J. Idar, R. A. Lucht, R. Scammon, J. W. Straight and C. B. Skidmore, "PBX 9501 High Explosive Violent Response/Low Amplitude Insult Project: Phase I," Report LA-13164-MS (1997), Los Alamos National Laboratory, Los Alamos, NM.

(2)S. K. Chidester, L. G. Green and C. G. Lee, "A Frictional Work Predictive Method For the Initiation of Solid High Explosives From LowPressure Impacts," Tenth International Detonation Symposium, , 1993, pp. 786-792.

(3)S. K. Chidester, C. M. Tarver and C. G. Lee, "Impact Ignition of New and Aged Solid Explosives," 1997 APS Topical Conference on Shock Compression of Condensed Matter, Amherst, MA, 1997.

(4)S. K. Chidester and C. M. Tarver, "Safety of Stockpile Aged Energetic Materials," 1998 Life Cycles of Energetic Materials, Fullerton, CA, March 29-April 1, 1998.

(5)R. A. Lucht and J. A. Charest, "Calibration and Use of a Rugged New Piezoresistive Transducer," 1995 APS Topical Conference on Shock Compression of Condensed Matter, Seattle, Washington, August 13-18, 1995, 1995, pp. 1041-1044. mechanically induced reaction, and accounts for the small changes in threshold velocity with density differences, and little or no changes in the threshold behavior as a function of $\mathrm{HE}$ thickness. Extrapolation of the threshold criterion curve to similar experiments will require scaling for differences in projectile, target, and $\mathrm{HE}$ material characteristics.

\section{ACKNOWLEDGEMENTS}

We gratefully acknowledge the programmatic and technical support and encouragement of our LANL colleagues Phil Howe, Gregory Buntain, George Hurley and Luis Salazar in these research efforts. We also thank Steven K. Chidester of Lawrence Livermore National Laboratory for his helpful suggestions regarding this research. The experimental results reported here have been a successful culmination of numerous individual efforts. Accordingly we would also like. to acknowledge their contributions to this project: JCI-NTS shop, the DX-5 designers and machinists, the DX-4 firing site support staff, the ESAWMM assembly crew and the ESA-EA technical staff involved with the various aspects of the experiments.

(6)D. S. Phillips, C. B. Skidmore., D. J. Idar, S. F. Son, R. B. Schwarz and B. W. Asay, "Defect Structures in Some Insulted HMX Composites," Inter/Micro 98, Chicago, Illinois, 10-14 August 1998.

(7)D. J. Idar and e. al., "Post-Test Characterization of Damaged PBX 9501 from Modified Steven Tests," in progress. (1998).

(8)B. F. Henson, R. K. Sander, S. F. Son, J. M. Robinson, P. M. Dickson and B. W. Asay, "Dynamic Measurement of the HMX b-d Phase Transition by Second Harmonic Generation," Physical Review Letters (May 1998).

(9)R. J. Scammon, R. V. Browning, J. Middleditch, J. K. Dienes, K. S. Haberman and J. G. Bennett, "Low Amplitude Insult Project: Structural Analysis and Prediction of Low Order Reaction," Eleventh International Detonation Symposium, Snowmass, Colorado, August 31-September 4, 1998, 1998, .

(10)B. W. Asay, P. M. Dickson, B. Henson, C. S. Fugard, D. J. Funk and D. J. Idar, "Dynamic Measurement Of The Influence Of Projectile Radius And Velocity On Strain Localization During Impact Of An Energetic Material," Eleventh International Detonation Symposium, Snowmass, Colorado, August 31-September 4, 1998, 1998, . 